\title{
CONSIDERACIONES DE LA FRAGILIDAD HUMANA \\ FRENTE A LA CONDUCTA MORAL DEL MEDICO
}

\author{
JUAN CARLOS ÁVILA MORALES
}

Médico magister en Salud Pública

Candidato a Doctor en Bioética.

Docente Asistente de la Facultad de Medicina de la Universidad Militar Nueva Granada

juan.avila@unimilitar.edu.co

Calle 44 No 67-17 Barrio Salitre Greco - Bogotá (Colombia)

Teléfono 6967704 - 3222439660

Recibido: 23 Marzo 2017 Aceptado: 30 Mayo 2017

\section{RESUMEN}

Este artículo hace parte de una investigación doctoral sobre el mal y la maleficencia en el acto médico, tratando en este escrito las consideraciones relacionadas con la fragilidad humana, su relación con el ejercicio de la medicina y el alcance de la conducta moral del médico. Mediante el método hermenéutico para la interpretación de los documentos encontrados se desarrolla un abordaje analítico de la temática encontrando que desde la ética filosófica se encuentra una gran diversidad de pensamientos bioéticos aplicados a la salud, basados en el respeto de toda persona humana. Es necesario considerar como parte del análisis bioético (en la relación médico paciente, la naturaleza de las decisiones y el conocimiento médico), los conceptos teorizados desde la filosofía, teniendo en cuenta que en el ejercicio de la medicina las valoraciones éticas pueden relacionarse con los dilemas surgidos en el encuentro de los principios y valores de la profesión frente a la autonomía y los deseos de los pacientes.

El desarrollo tecnológico y del conocimiento que componen la ciencia (episteme) y el arte (techne) de la medicina, junto a los cambios en la concepción de la relación médico paciente y en las dimensiones del acto médico (antropológica, disciplinar y determinantes sociales) han propiciado la indiferencia del profesional frente al sufrimiento, el dolor, la fragilidad y la vulnerabilidad de las personas (pacientes). Es necesario que el medico reflexione sobre los conceptos de compasión, solidaridad y responsabilidad con el otro, para tratar de restaurar la salud y sanar al enfermo y cuando no lo logre, pueda ayudar y cuidar en cuanto a su dolor y vulnerabilidad. Esto podría fundamentar el ejercicio de la profesión entre otras consideraciones en el imperativo kantiano, en la medida que el profesional de la salud actúe de tal manera que la máxima de su acción pueda ser querida como una máxima universal.

Palabras clave: Bioética; Fragilidad; Moral; Deshumanización; Medicina general. 
Ávila J.

\title{
CONSIDERATIONS OF HUMAN FRAGILITY \\ FRONT OF THE MORAL MEDICAL CONDUCT
}

\begin{abstract}
This article makes part of a doctoral research about evil and maleficence in the medical act, attending in this text the moral considerations around the medical practice, which include aspects related with human fragility, it's relationship with the medical praxis and the doctor's moral demeanor reach. By hermeneutical method for the interpretation of the found documents an analytical approach is made about the topic, finding that from the point of view of philosophical ethics, there is a great diversity of bioethic thoughts applied in health, based on respect for every human being. It is necessary to consider as a part of the bioethic analysis (around the doctor and patient relationship, the decisions' nature and the medical knowledge) the concepts theorized from philosophy, taking into account that in the practice of medicine the ethical reviews, can be related with the ethical struggles emerged at the meeting of principles and values of medicine versus the autonomy and desires of the patients.

The techological and knwoledge developments that encompasses science (episteme) and art (techne) of medicine, joined with the changes in the view of the relationship between patient and doctor and the dimensions of the medical act (anthropological, discipline and social determinants) have led to the professional's indifference in front of suffering, pain, fragility and vulnerability of people (patients). It is necessary that the doctor ponder about the concepts of compassion, solidarity and responsibility among the others, to try to reestablish health and to heal the ill, and even if can't be done, the doctor might help and care in matters of the patient's pain and vulnerability. This could base the exercise of the profession among other considerations in the Kantian imperative, to the extent that the health professional to act in such a way that the maxim of the action can be willed as a maximum universal.
\end{abstract}

Keywords: Bioethics; Fragility; Moral; Dehumanization; General medicine. 


\section{CONSIDERAÇÕES DA FRAGILIDADE HUMANA FRENTE À CONDUTA MÉDICA MORAL}

\section{RESUMO}

Este artigo faz parte de uma investigação de doutorado sobre maldade e maleficência no ato médico, acompanhando neste texto as considerações morais em torno da prática médica, que incluem aspectos relacionados à fragilidade humana, é relação com a práxis médica e o alcance moral do médico. Por meio do método hermenêutico para a interpretação dos documentos encontrados, faz-se uma abordagem analítica sobre o tema, descobrindo que, do ponto de vista da ética filosófica, existe uma grande diversidade de pensamentos bioéticos aplicados na saúde, com base no respeito a cada ser humano. É necessário considerar como parte da análise bioética (em torno do relacionamento médico e paciente, a natureza das decisões e o conhecimento médico) os conceitos teorizados da filosofia, levando em conta que na prática da medicina as avaliações éticas podem ser relacionados com as lutas éticas surgiram no encontro dos princípios e valores da medicina versus a autonomia e os desejos dos pacientes.

Os desenvolvimentos tecnológicos e de conhecimento que abrangem ciência (episteme) e arte (techne) da medicina, unidos com as mudanças na visão da relação entre paciente e médico e as dimensões do ato médico (determinantes antropológicos, disciplinares e sociais) levaram à indiferença do profissional diante do sofrimento, dor, fragilidade $e$ vulnerabilidade das pessoas (pacientes). É necessário que o médico considere os conceitos de compaixão, solidariedade e responsabilidade entre os outros, para tentar restabelecer a saúde e curar os doentes e, mesmo que não possa ser feito, o médico pode ajudar e cuidar em matéria de Dor e vulnerabilidade do paciente. Isso poderia basear o exercício da profissão entre outras considerações no imperativo kantiano, na medida em que o profissional de saúde atua de tal forma que a máxima da ação possa ser considerada como máxima universal.

Palavras-chave: Bioética; Fragilidade; Moral; Desumanização; Medicina geral. 


\section{Introducción}

La relación médico-paciente se encuentra enmarcada en las reglas morales de la confidencialidad, veracidad y consentimiento informado, acorde a los preceptos establecidos en los principios de la Bioética (autonomía, beneficencia, justicia y no maleficencia). En esta relación y pese a la preponderancia de la autonomía siempre está implícita una relación de jerarquía, que ha llevado a una concepción errónea bidireccional de sumisión-poder, lo cual puede potencialmente disminuir la posibilidad de cumplir el fin último del ejercicio médico: la salud integral y calidad de vida del paciente.

Es en este contexto en que la actitud indiferente adolece y ahonda la fragilidad propia del ser humano, especialmente cuando este se encuentra debilitado por un estado de afección física o psicológica. La indiferencia ante la sensación de desequilibrio fisiológico silencia las experiencias de los pacientes y el significado personal que en ellos acarrea. Es por ello que a partir de las revisiones y reflexiones de los aspectos del mal, la maleficencia y la deshumanización en documentos previos de la presente investigación, se procede a indagar y puntualizar los tópicos relacionados con la fragilidad humana, su relación con el ejercicio de la medicina y el alcance del concepto de la conducta moral del médico.

El análisis y entendimiento de estos aspectos circunscritos al desarrollo de la práctica médica, son necesarios para poder hacer un abordaje de la teoría del mal en la medicina desde una perspectiva bioética, ya que en el ejercicio y la práctica del médico hay que considerar que toda valoración de tipo ético sobre la bondad o la maldad del actuar humano concreto, debe hacerse a la luz de sistema de referencia que pueda ser aplicado al ejercicio de la profesión.

\section{Metodología $^{1}$}

La recolección de datos y el uso de las fuentes de información se hicieron por medio del método hermenéutico para el análisis e interpretación de los textos. Para el presente escrito se realizó la búsqueda en la base de datos Pubmed, EbscoHost, Dialnet, ProQuest, Bireme, Elsevier y ScienceDirect con las palabras: mal, maleficencia, practica, médico, medicina, fragilidad, moral, conducta, iatrogenia, mala práctica, indiferencia, solidaridad; se utilizaron diversas combinaciones de los mismos con e l conector "Y". La búsqueda se amplía utilizando los términos en inglés: evil, maleficence, practice, doctor, medicine, fragility, moral, conduct, iatrogenia, malpractice, indifference, solidarity. Se incluyeron aquellos artículos que estuvieran en texto completo y se descartaron los escritos correspondientes a editoriales y cartas al editor y se tomó como criterio de búsqueda desde el año 2000 al 2016, dando prioridad a los artículos de los años más recientes.

La búsqueda bibliográfica también incluyó libros que cumplieron con los criterios de búsqueda y representaban un aporte importante para la construcción del presente escrito, para lo cual se amplió el rango de tiempo y por su importancia para el desarrollo de la investigación.

\section{El concepto de la fragilidad humana y su rela- ción con el ejercicio de la medicina}

De acuerdo al diccionario etimológico, el término fragilidad proviene del latín fragilis, que significa quebrar o romper. Desde el punto de vista biomédico, la fragilidad es conceptualizada como un estado de pérdida funcional, que hace a la persona más susceptible o propensa a sufrir eventos adversos en salud, como caídas, hospitalizaciones y/o disminución de la independencia por discapacidad. Dicho estado se presenta cuando la capacidad de reserva fisiológica de los diferentes órganos del cuerpo se ve reducida por procesos tales como el envejecimiento, en el que pequeñas alteraciones orgánicas desencadenan una serie de complicaciones graves que pueden conllevar incluso la muerte (1).

Por otra parte, desde una perspectiva antropológica, la fragilidad se define como aquella condición de finitud propia del hombre, que lo lleva a concebirse a sí mismo como un ser indefenso y susceptible de sufrir daño. Así, la fragilidad se mide y va ligada al punto de ruptura y también se asocia al concepto de dependencia (2). Es una condición permanente del ser humano que se caracteriza por la limitación y carencia de algo en todas las expresiones y ámbitos de su corporalidad (3); pues el cuerpo es limitado, y es por ello que las expectativas se ven truncadas por las carencias del cuerpo. Al fin y al cabo, el cuerpo es posibilidad, pero también es límite, y la corporalidad siempre aparece como tal.

1. El presente artículo hace parte de una investigación cuya tesis fue aprobada para la obtención de un título de Doctorado. La investigación incluye la revisión documental del concepto y alcance de las consideraciones morales de la maleficencia en torno a la práctica médica, dentro de los cuales se incluyeron los tópicos relacionados con la fragilidad humana, conducta moral del médico, iatrogenia y mala práctica, mal y maleficencia, indiferencia y solidaridad. 
La inmensa riqueza del ser se acompaña de la debilidad congénita de la naturaleza humana. Esta condición de vulnerabilidad se hace más evidente cuando se atraviesa un estado de enfermedad (que nos vuelve frágiles), que irremediablemente acarrea consigo dolor y sufrimiento $e$ incluso puede terminar en la muerte (2). Cuando el ser humano se encuentra acorralado por el sufrimiento, reflexiona sobre el dolor que padece y sobre la naturaleza de su vida, ya que a medida que aumenta la amenaza se incrementa la duda sobre la validez de los valores (4) y es entonces cuando admite la finitud propia de su existencia y se concientiza de su fragilidad (5). La fragilidad tiene que ver entonces con la posibilidad de padecer una enfermedad, de sufrir, y morir. Es lo que condiciona nuestra vida a la extinción biológica y biográfica y pone un límite a nuestras posibilidades, proyectos y anhelos (6).

Los términos fragilidad y vulnerabilidad son intercambiables, ya que en ambos casos se comparte la noción de que existe cierta limitación y ausencia de algo (3). Esto cobra importancia cuando la fragilidad del hombre trasciende su dimensión biológica y se empieza a analizar desde una perspectiva social, en la que se concibe al humano como un ser susceptible a sufrir daños por las condiciones ambientales y socio-económicas en las que se desenvuelve. Tales condiciones son, entre otras: marginación, exclusión social y pobreza. La vida del ser humano es extremadamente vulnerable cuando se presentan situaciones socio-económicas desfavorables, como bajos ingresos económicos, que irremediablemente condicionan a la persona a padecer de hambre, desamparo social $e$ indigencia. Las carencias alimenticias, las condiciones de vida insalubres y la indefensión ante el dolor, conllevan daño físico, que a su vez predispone a la persona para el desarrollo de enfermedades. El ser humano malnutrido se vuelve frágil, pues no solo sus funciones orgánicas se ven comprometidas, sino que además sus habilidades cognitivas, necesarias para desempeñar bien un trabajo, también se ven deterioradas (7).

La fragilidad antropológica, al ser entendida como un estado en el que el ser humano es permanente susceptible a la destrucción y finitud de su existencia, puede verse magnificada en función de los factores medio-ambientales y socio-económicos en los que la persona se desenvuelve. Los médicos y demás profesionales de la salud están en la obligación moral de reconocer la fragilidad del ser humano en todas estas dimensiones. El médico, por medio de una "actitud de cuidado", debe ser sensible ante el sufrimiento y las necesidades de sus pacientes, y así mismo, está en el deber de ser solidario y de demostrar un sentido de preocupación y responsabilidad por el otro ser humano (6). Finalmente, tiene el imperativo moral de defender los derechos de sus pacientes y propender para que todas las personas, independientemente de su condición social y económica, reciban atención médica oportuna y de calidad (8).

La vulnerabilidad no solo constituye el fundamento del autocuidado y su razón de ser, sino que alude a un rasgo del ser humano relacionado con el imperativo ético de velar por la protección de la vida y su respeto; al tiempo que su noción conserva una relación muy estrecha con los principios éticos de autonomía, dignidad e integridad. Esta vulnerabilidad adquiere formas y tipologías distintas según grupos e individualidades, y de este modo existen diversos tipos fundamentales de clasificarla: Ontológica (tiene su raíz en el ser humano), somática (propia y específica del cuerpo, del ser encarnado), psicológica (relacionada con la psique y con una realidad vulnerable, que se puede enfermar), social (en el ser político y la apertura al otro) y espiritual (sentido de la existencia) (9).

Por otra parte, una ética de la fragilidad invita a considerar la humanidad más allá o más acá de la autonomía de la voluntad (10) ya que el principio de autonomía estará circunscrito dentro de los límites que le establecen los principios de dignidad y vulnerabilidad, que así mismo pueden relativizar la libertad del paciente (11). En el contexto de una persona frágil, que debilitada por su condición de enfermedad confía en los conocimientos del médico para recobrar la salud, tener alivio a sus dolencias o ser acompañada en el buen morir, la humanización de la salud hace alusión a reconocer al paciente como otro ser humano, con derechos que deben ser respetados. Entre sus derechos está que se le provea atención adecuada y oportuna, ser tratado con respeto y reconocido como una persona digna de ser asistida en todas sus dimensiones y necesidades. Esta dignificación del paciente en estado de fragilidad es el objetivo y fin de la humanización de la salud (8).

Más allá de la relación médico-paciente, esta humanización también implica hacer más humanas las instituciones de la salud, en las que no solo se busca defender los valores esenciales del ser humano, sino que además se debe favorecer la calidad de los servicios de salud de forma igualitaria. cuando existen desigualdades de poder, conocimientos o recursos económicos, los más fuertes están en la obligación moral de respetar y proteger la fragilidad y vulnerabilidad del otro (12) y de acuerdo con Ricoeur, la medicina, mediante su humanización debe 
buscar y defender las necesidades de los pacientes o sus "intereses vitales" desde un enfoque de justicia social. Este concepto de justicia, por su parte, es la base de algunas de las posiciones más actuales en la bioética latinoamericana, a partir del precepto del autor de que hace falta "una lógica de la sobreabundancia que efectivamente nos impulse a hacer justicia a favor de los otros, aplastados por un domino tan terrible que ni siquiera explota, sino que margina" (13).

\section{El alcance de la conducta moral del médico}

Para abordar este tema se debe empezar por definir qué es la moral, pues esta puede ser entendida como el conjunto de normas y códigos de acción impuestos en una sociedad que regula la conducta de los individuos que la conforman (14). La moral "designa el conducto de principios, imperativos o ideas morales de una época o una sociedad dada", y es un sinónimo de humano en tanto intervienen las facultades conscientes del hombre, la razón y la voluntad (15). La moralidad, por su parte, hace alusión al sistema moral que las personas adoptan para afrontar dilemas y/o hacer sus propios juicios morales. Puede ser concebida también como una guía de comportamiento que los seres racionales utilizan para gobernar su actuar y el actuar de otros (16). Entretanto, el acto o la conducta moral se ha descrito como un acto humano que se realiza libre y conscientemente y que se conforma (o no) con el fin último del hombre o con la norma objetiva de moralidad. El acto moral (15) tiene las siguientes propiedades:

- Responsabilidad: Definida como la cualidad mediante la cual el autor de un acto moral debe responder ante una autoridad superior.

- Mérito o Demérito: Valor moral de un acto que le hace merecedor de un premio o un castigo.

La medicina es sin duda alguna una actividad con un profundo carácter moral, es una "empresa moral" que según una serie de cánones y creencias definen cómo debe ser la conducta médica (17). El Juramento Hipocrático, considerado el principal modelo de moralidad médica, imparte un conjunto de obligaciones morales que, idealmente, deben ser acogidas por aquellos que practican el arte de la medicina. Es de esperarse entonces que todo acto médico sea un acto moral, ya que ocurre entre dos personas que de manera libre consienten que una de ellas le otorgue confianza a la otra persona con el fin de recuperar la salud o, en su defecto, obtener alivio a sus dolencias (18). El acto médico es entonces una acción interpersonal, donde la moral supera todo conocimiento y técnica. Es de carácter personal, bilateral, moral y con conciencia ética, en el que se crea una relación entre el enfermo que busca ser sanado y el médico que ha realizado un juramento, para procurar que el/la paciente tenga mejor calidad de vida (19).

El carácter moral de la conducta médica influye en múltiples aspectos del ejercicio de la medicina, existiendo aspectos fundamentales que hacen de la medicina una actividad con un estatus moral especial (20), en los que la conducta moral del médico tiene una repercusión directa:

La relación médico-paciente: Esta relación se caracteriza por ser asimétrica y vertical. El médico está en el polo superior con un rol activo y autoritario, mientras que el paciente se encuentra en el polo inferior, debido a su estado de enfermedad (21). Esta condición, sumada al hecho de que el paciente carece de la información necesaria para responder preguntas fundamentales sobre lo que le sucede y sobre cuál es la conducta a seguir, hacen que el enfermo pierda en cierta medida la libertad para tomar sus propias decisiones y tenga que recurrir a alguien más que posee el conocimiento para que decida por él o ella qué es lo más benéfico.

Esta dependencia que el paciente tiene del médico lo coloca en una posición vulnerable. Es así entonces como la relación médico-paciente ha sido descrita como "una relación desigual basada en promesas y en vulnerabilidad" (17). Esta condición de fragilidad imparte en el profesional de salud una serie de obligaciones morales para con su paciente, ya que la persona en estado de enfermedad debe ser tratada con compasión. Una verdadera acción sanadora requiere de la compasión como requisito moral, que le permitirá al médico entender lo que significa la enfermedad para el paciente, y en esa medida tomar decisiones que verdaderamente lo favorezcan. Asimismo, le permitirá brindar información médica de manera clara y veraz al paciente o a su representante legal, sin ser ocultada, manipulada o revelada parcialmente, sobre su condición de salud y sobre las opciones terapéuticas, pues este es un imperativo moral en la relación médico-paciente.

Otra de las exigencias morales en la relación médico-paciente es la de obtener el debido consentimiento informado para la realización de tratamiento.

La naturaleza de las decisiones médicas: Los médicos en el curso de su ejercicio profesional se enfrentan 
diariamente a situaciones que no solo requieren de un alto conocimiento técnico-científico, sino que además lo confrontan con toda una serie de dilemas éticos que van desde el aborto, el cuidado de un menor con deformidades múltiples, la reproducción asistida o prolongación de la vida, por solo por nombrar algunos. En cada uno de estos diferentes contextos el médico debe, mediante el ejercicio de una conducta moral, tomar decisiones que propendan por el bien de su paciente.

$\mathrm{Al}$ reconocerse a sí mismo como un agente moral, el médico tiene en todo momento que evaluar el estatus ético de las recomendaciones terapéuticas que realiza teniendo en cuenta que el propósito y fin último de sus intervenciones es el bienestar de la persona a la que está tratando. Sin embargo, este proceso no es del todo sencillo, ya que aquello sobre lo que el médico tiene que deliberar y adoptar una conducta, es la vida misma de seres humanos. Es entonces cuando conceptos como dignidad humana y calidad de vida entran en contexto. Adicionalmente, en una sociedad moralmente pluralista, un mismo acto médico puede ser catalogado como moralmente correcto por algunos, mientras para otros es categóricamente inmoral (tal es el caso de la eutanasia).

Frente a la insensatez, el egoísmo y la mala fe, el médico se enfrenta a tomar dos decisiones relacionadas con la razón y la cura del alma. En primera instancia puede volverse "indignado", lo que lo lleva a no tener en cuenta la razón del paciente o apelar a toda la razón del enfermo y contentarla. En segunda instancia, se enfrenta a darle menor valor, perder las posibilidades científicas o apoyarse en al antiguo precepto basado en la ciencia y la humanidad, y esta idea necesita una constante renovación (22).

El conocimiento médico: El concepto universal de método, vinculado al concepto moderno de ciencia, es una forma constructiva de pensar y se puede afirmar que el método construye el objeto de conocimiento. En el caso del médico, su ciencia y su práctica se desarrollan en la tenue línea que media entre el terreno del conocimiento (propio de las ciencias naturales y el raciocinio en el acontecer natural) y la confrontación con los enigmas y dilemas mentales y espirituales (23).

A este respecto, la conducta moral del médico también se ejerce en el aprendizaje y maestría de su ciencia y arte. Aquellos profesionales privilegiados que entran a ser miembros de la comunidad médica están en la obligación moral de utilizar los conocimientos que les han sido confiados por sus maestros para el cuidado de los enfer- mos. Asimismo, al entrar a conformar esta comunidad, los profesionales tienen el imperativo moral de preservar, enseñar y transmitir dicho conocimiento (20). La idea moderna de la enfermedad se ve como un proceso natural, tratando de curar al cuerpo o que él mismo evolucione a un proceso más favorable, donde la terapia médica se fundamenta en el conocimiento científico y la humanidad. Tanto médico y paciente deben ser razonables para afrontar juntos este proceso natural en el que el médico pone ante el paciente su saber, pericia e instrucción (24).

\section{Discusión}

El medico científico ha reemplazado, en su concepto y competencia, a los médicos hipocrático y medieval, pero conserva, como características que los unen, el ser eficaz, lógico e insensato. Frente a la enfermedad, el médico se une al paciente, le instruye y aplica una terapia razonable, que bien puede ser aceptada o rechazada por el paciente. De cualquier modo, la acción médica resultante se sustenta en dos columnas, por un lado, se encuentra el conocimiento científico y la capacidad técnica, o en otras palabras la episteme (ciencia y metafísica) y la techne (praxis y arte). Por otro lado, la ética de la humanidad (dignidad, autonomía y valor del individuo) (22).

La dignidad del ser humano ha de contar con dos aspectos fundamentales de la vida y dados por la autonomía (correlacionada esta con las exigencias de justicia): la fragilidad y la vulnerabilidad (correlacionadas con la justicia y el cuidado). Ahora bien, para que el concepto de moral no quede reducido a las cuestiones de justicia, Kohlberg, teniendo en cuenta a la ética de la preocupación de Gilligan, une las exigencias de la justicia con la preocupación por el bien de los otros a través de la compasión y el amor, es decir, une justicia y benevolencia (25).

Como se ha podido ver, la ética filosófica ha inspirado una gran diversidad de pensamientos bioéticos aplicados a la salud, en la que el concepto de la naturaleza humana forma parte de todo acto ético. La moral común es el comportamiento regido por costumbres y pautas culturales de una sociedad en la que el pensamiento ético evoluciona desde el monólogo interior (ética de conciencia) hacia la relación con el otro (ética de responsabilidad). Por lo tanto, se exige actuar en respeto de toda persona humana, que es siempre un fin y no solo un medio (26).

El mal no necesita proceder de una esencialidad que pueda dar una respuesta racional abstracta a lo qué es, sino que el mal es cuando se encarna tiene rostro e identidad, 
se hace presente (es un nombre) ya es algo menos imposible y se convierte en una interpretación existencial del dolor y la deshumanización (27). La naturaleza humana había sido asumida como buena en sí misma y capaz solo de manera accidental de realizar una acción mala y por lo tanto la causa de dicha acción se buscaba en lo otro distinto de lo propiamente humano. La condición moral presente del hombre se encuentra determinada por la legalidad propia de la ley moral, el deseo y el albedrio; si el bien moral consiste en la subordinación del deseo a la ley moral, el mal moral consistirá en la inversión de la subordinación de estos elementos a la acción. Es por ello que el reto en el análisis de la perversidad humana consiste en explicar cómo es posible que se produzca esta inversión del orden moral de los motivos de la acción (28).

En el ejercicio de la medicina hay que considerar que toda valoración de tipo ético sobre la bondad o la maldad del actuar humano concreto, debe hacerse a la luz de un determinado sistema de referencia, en el que los conflictos éticos surgen cuando los valores de la medicina van en contravía con los deseos de los pacientes. Cuando el profesional de la salud cumple la indicación dada sin tener en cuenta su imperativo hipocrático y lo supedita a un imperativo económico, puede dejar de lado la toma de la decisión que iría en bien del paciente y la cambia por otra, que si bien no tiene la intencionalidad de provocar el daño, podría provocarlo en el paciente. Esto no implica que el profesional sea consciente del mal implícito en la acción, pero en ausencia del diálogo consigo mismo, no puede reconocerse moralmente responsable y mostrar arrepentimiento, porque su deber había sido, en su momento y retrospectivamente, cumplir con la ley sin cuestionarla (29).

\section{Conclusiones}

El medico ha de poseer cualidades y valores que le permitan establecer una relación de confianza con el paciente, eligiendo acciones acordes a los fines de la medicina (restaurar la salud y sanar al enfermo) y cuando no pueda, ayudar y cuidar al enfermo en su dolor y vulnerabilidad. La condición fundamental para alcanzar una conducta medica ideal están enmarcadas por la humanidad, discernimiento, confiabilidad (fidelidad al voto de confianza), justicia, fortaleza, templanza, desapego de uno mismo, honestidad, empatía, respeto, profesionalidad y objetividad (30).

La medicina se compone de la ciencia (episteme) y el arte (techne) y por lo tanto los principios rectores de la práctica (praxis) médica estarán dados por el conocimiento (científico y tecnológico) y la ética de humanidad. El acto médico es el resultante del equilibrio de tres dimensiones que abarcan desde lo antropológico al tener en su esencia al hombre (tanto el profesional como el paciente), lo disciplinar entendido por la formación y el ejercicio profesional y finalmente la dimensión dada por el contexto social en la que la praxis está regulada por determinantes científicos técnicos, económicos, administrativos, sociales y políticos (31).

El médico debe estar inmerso en la relación con el paciente siendo imperativo el recuperar el principio de humanidad, debido a que le enfermedad provoca sufrimiento, dolor y aflicción para el ser, y en algunas ocasiones se cae en la indiferencia al sufrimiento, el dolor, la fragilidad y la vulnerabilidad, debido a que, en los procesos de formación del médico, en el abordaje de estos tópicos no hay realidades epistemológicas ya se aprende un saber sin la comprensión del saber.

Desde la Bioética el concepto y alcance de la solidaridad está presente en los lineamientos internacionales para fomentarla entre los seres humanos con la cooperación internacional para tal efecto y en el que "los Estados deberían respetar y fomentar la solidaridad entre ellos y deberían también promoverla con y entre individuos, familias, grupos y comunidades, en particular con los que son más vulnerables a causa de enfermedades, discapacidades u otros factores personales, sociales o ambientales, y con los que poseen recursos más limitados" (32).

El concepto del mal ha sido asumido desde lo teológico (con relación al pecado), desde lo moral (Kant) y desde lo político (Arendt), aparece relacionado con el acto y la acción confluyendo en una resultante dada por el daño al paciente, en la cual se sobreponen los conceptos de iatrogenia y mala práctica. Es necesario tener en cuenta el efecto telefístico en la salud ya que en muchas de sus actividades, intervenciones y procedimientos es necesario realizar un mal para hacer un bien por lo tanto se hace necesario hacer un abordaje de la teoría del mal en medicina desde una perspectiva bioética.

Es necesario que el medico siga asumiendo los conceptos de compasión, solidaridad, responsabilidad del otro y que fundamente el ejercicio de la profesión en el imperativo kantiano en la medida que actúe de tal manera que la máxima de su acción pueda ser querida como una máxima universal.

\section{Conflicto de intereses Ninguno}




\section{Referencias}

1. Collard R, Comijs H, Naarding P Voshaar O. Physical frailty: Vulnerability of patients suffering from late-life depression. Aging and Mental Health. 2014; 18 (5): 570-578.

2. Tomás y Garrido G. La Salud. Una Perspectiva Bioética. Revista de Fisioterapia. 2013. 0: 11-13.

3. Rosas C. (2015). La Vulnerabilidad Humana: Un freno a la Autonomía. Revista de Bioética Latinoamericana. 16: 1-16.

4. Jünger E. Sobre el dolor. En: Jünger E. Sobre el dolor. Barcelona: Libergraf; 1995. p. 9-85.

5. Selli L. Cuidados ante el dolor y el sufrimiento. En: Tealdi. J. Diccionario Latinoamericano de Bioética. Bogotá: UNESCO - Red Latinoamericana y del Caribe de Bioética: Universidad Nacional de Colombia; 2008. p. 62-63.

6. Feito L. Vulnerabilidad. Anales del Sistema Sanitario de Navarra. 2007; 30 (3): 7-22.

7. Rosanelli C, Cardoso A, De Assis L, Da Silva T, De Siqueira J. La Fragilidad Humana frente a la Pobreza y el Hambre. Rev. Bioét. 2015; 23 (1): 90-98.

8. Andino C. Bioética y Humanización de los Servicios Asistenciales en la Salud. Revista Colombiana de Bioética. 2015; 10 (1): 38-64.

9. Torralba F. El cuidado de uno mismo. Perspectiva ética. En: Sandrin L, Calduch-Benages N, Torralba F. Cuidarse a sí mismo. Para ayudar sin quemarse. Madrid: Editorial y distribuidora S.A; 2007.p. 77-103.

10. Moreno A. La ética de la vulnerabilidad de Corine Pelluchon. Revista Internacional de Filosofía. 2013; 58: 171-178.

11. Velásquez L. La relación médico-paciente: Una aproximación al problema de la autonomía. Revista Colombiana de Bioética. 2012; 7(1): 163-171.

12. Thomasma, D. The Vulnerability of the Sick. Bioethics Forum. 2000; 16 (2): 5-12.

13. León F. Justicia y bioética ante las desigualdades en salud en Latinoamérica. Revista Colombiana de Bioética. 2010; 5(1): 85-99.

14. Rodríguez M. Sobre ética y Moral. Revista Digital Universitaria. 2005; 6(3): 1-5.

15. Agudelo G. Ética Profesional de la Educación. Madrid: Ediciones Verdad y Vida; 1989.

16. Gert B, Culver C, Clouser K. Bioethics: A Return to the Fundamentals. Oxford: University Press; 1997.

17. Pellegrino E. Toward a Reconstruction of Medical Morality. The American Journal of Bioethics. 2006; 6(2): 65-71.
18. Porter K, Villalobos J, Tarasco M. Introducción a la Bioética. México: Méndez Editores; 2009.

19. Reyes P, Delong R. Algunos dilemas éticos en la práctica médica. Archivos de Cardiología de México. 2010; 80(4): 338-342.

20. Pellegrino E. The Medical Profession as a Moral Community. New York Academy of Medicine. 1990; 66 (3): 221-232.

21. Gracia D. Ética y Vida No. 2: Bioética Clínica. Bogotá: Editorial El Búho; 2001.

22. Jaspers K. La idea del médico. En: Jaspers K. La práctica médica en la era tecnológica. Barcelona: Editorial Gedisa Barcelona; 1998.p. 9-26.

23. Gadamer H. Hermenéutica y psiquiatría. En: Gadamer H. El estado oculto de la salud. Barcelona: Editorial Gedisa Barcelona; 2001.p. 177-187.

24. Jaspers K. Médico y paciente. En Jaspers K. La práctica médica en la era tecnológica. Barcelona: Editorial Gedisa Barcelona; 1998.p. 27-56.

25. Conill J, Cortina A. La fragilidad y la vulnerabilidad como partes constitutivas del ser humano. En: Beca J. Astete C. Bioética clínica. Santiago de Chile: Mediterráneo; 2012. p. 117-127. (48)

26. Kottow M. Fundamentos de ética. En: Kottow M. Introducción a la bioética. Santiago de Chile: Editorial Mediterráneo; 2005. p. 17-59. (52)

27. Fernández J. Violencia y resentimiento. Jean Améry o el humanismo inflexible Revista de Filosofía. 2006; 37 : 23-36. (53)

28. Cardona L. El reconocimiento dialógico de la perversidad humana. En: Cardona L. Mal y sufrimiento humano. Un acercamiento filosófico a un problema clásico. Bogotá: Editorial Pontificia Universidad Javeriana; 2013. p. 251-331. (54)

29. Kottow M. Maleficencia y la banalidad del mal: una reflexión bioética. Revista latinoamericana de Bioética. 2014; 14(1): 38-47. (54)

30. Rodríguez E. Bioética clínica: Modelos de relación clínica. En: León F, Arratia A, Casas M, Rodríguez E. Bioética general y clínica. Santiago de Chile: Fundación interamericana ciencia y vida; 2010. p. 147-174. (55)

31. Ávila-Morales JC. La deshumanización en medicina. Desde la formación al ejercicio profesional. Iatreia. 2017 AbrJun;30(2):216-29. DOI 10.17533/udea.iatreia. v30n2a11.

32. UNESCO. Declaración Universal sobre Bioética y Derechos Humanos. Revista Latinoamericana de Bioética; 2006: 118-157. 